\title{
Automation Technologies Impact on the Work Process of Nuclear Power Plants
}

Ahmad Y Al Rashdan, Torrey J Mortenson

September 2018

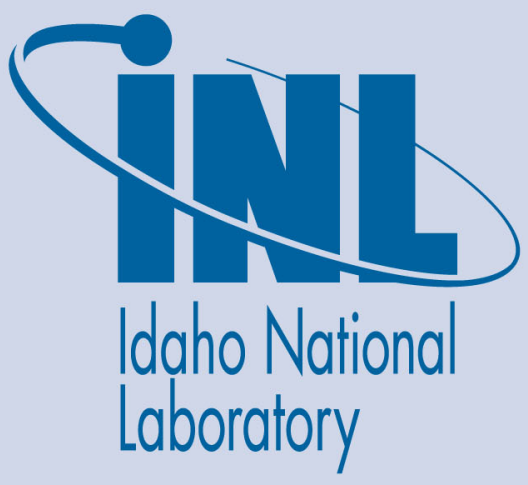

The INL is a U.S. Department of Energy National Laboratory operated by Battelle Energy Alliance 


\title{
Automation Technologies Impact on the Work Process of Nuclear Power Plants
}

\author{
Ahmad Y Al Rashdan, Torrey J Mortenson
}

September 2018

Idaho National Laboratory Idaho Falls, Idaho 83415

http://www.inl.gov

Prepared for the

U.S. Department of Energy

Under DOE Idaho Operations Office

Contract DE-AC07-05ID14517 


\section{Light Water Reactor Sustainability Program}

\section{Automation Technologies Impact on the Work Process of Nuclear Power Plants}

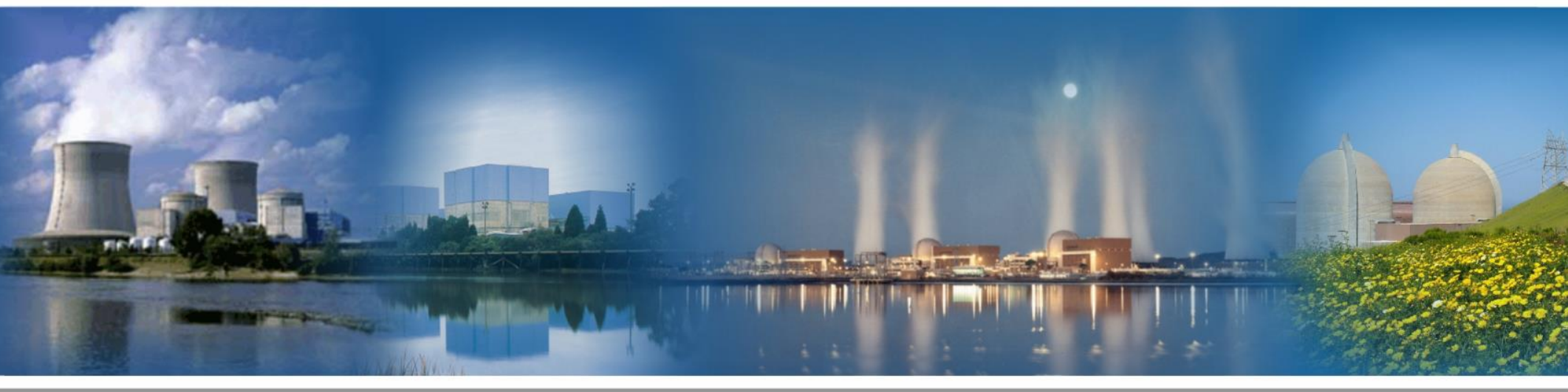

September 2018

U.S. Department of Energy

Office of Nuclear Energy 


\section{DISCLAIMER}

This information was prepared as an account of work sponsored by an agency of the U.S. Government. Neither the U.S. Government nor any agency thereof, nor any of their employees, makes any warranty, expressed or implied, or assumes any legal liability or responsibility for the accuracy, completeness, or usefulness, of any information, apparatus, product, or process disclosed, or represents that its use would not infringe privately owned rights. References herein to any specific commercial product, process, or service by trade name, trade mark, manufacturer, or otherwise, does not necessarily constitute or imply its endorsement, recommendation, or favoring by the U.S. Government or any agency thereof. The views and opinions of authors expressed herein do not necessarily state or reflect those of the U.S. Government or any agency thereof. 


\title{
Automation Technologies Impact on the Work Process of Nuclear Power Plants
}

\author{
Ahmad Al Rashdan and Torrey Mortenson
}

September 2018

Prepared for the U.S. Department of Energy Office of Nuclear Energy 



\section{ABSTRACT}

Nuclear power plants (NPPs) have been experiencing significant cost challenges to remain competitive with other power industries. This places a large burden on NPPs to minimize costs while sustaining excellent safety records. The Light Water Reactor Sustainability Program is a research and development program sponsored by the United States Department of Energy that supports the industry in overcoming this challenge. One of the main focus areas of the program is plant modernization, with automation as one of the key elements of modernization.

NPPs costs are distributed primarily across operations and maintenance activities in the plant. Regulatory burdens, increased market costs for skilled labor, and increased surveillance and maintenance activities in pursuance of extended life cycles of the nuclear fleet are among the main cost drivers at play. Work processes at NPPs involve many reviews and layers of approval to ensure safe execution of operation and maintenance tasks. The work process is burdensome for maintenance activities and involves some disparate processes, systems, and departments across the organization. These activities add costs due to inefficiencies and manual processes. This makes the work process a great candidate for automation technologies.

This work was performed to assess various automation technologies and the means by which they assimilate into the work process in order to make cost savings in NPPs. This report provides a large, but not exhaustive, list of relevant automation technologies at various states of maturity. The goal was to identify key informational items that would be useful to NPPs that are undertaking the effort to reduce costs through automation by identifying technology maturity levels, recent findings, and expected cost savings. Current deployment of automation technologies at NPPs exists on varying levels, so the information presented is aimed to give stakeholders the necessary information, regardless of their current technological status.

The results of this effort generated a list of technologies that fit each of three cost-saving categories. The technologies that have high cost-saving impact are identified as drones, electronic work packages, mobile devices, plant data integration, smart equipment, smart scheduling, wireless networks, wireless sensors, work data mining, and work risk models. The technologies that have medium cost-saving impact are identified as augmented reality, image information extraction, mobile equipment evaluation tools, radio-frequency identification, spatial mapping, three-dimensional animation, video recording, video monitoring, virtual reality, and wireless actuators. The technologies that have low cost-saving impact are identified as bar codes, electronic tags, image anomaly detection, image objects recognition, global positioning systems, interactive audio, motion recognition, smart tools, three-dimensional printing, video communication tools, Wi-Fi positing, and wireless beacons.

The effort concluded that technologies with high cost impact that requires more development and/or research into the technology or specific aspects of its application are drones, electronic work packages, plant data integration methods, smart equipment, smart scheduling, wireless sensors, work data mining, and work risk models. The technologies with medium cost impact that require more development and/or research into the technology or specific aspects of its 
application are augmented reality, image information extraction, mobile equipment evaluation tools, radio-frequency identification, virtual reality, and wireless actuators. The technologies with low cost impact that require more development and/or research into the technology or specific aspects of its application are image anomaly detection, image objects recognition, interactive audio, motion recognition, smart tools, and Wi-Fi positing. While the focus of this study is cost saving, these technologies have benefits beyond simply cutting costs, because many will also allow for efficiency gains and improvements in safety. 


\section{CONTENTS}

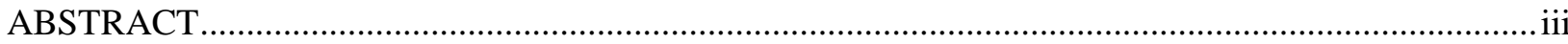

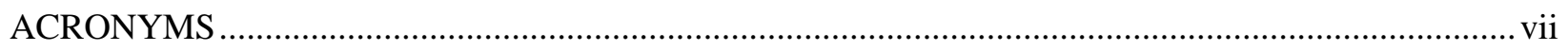

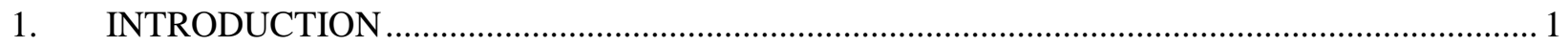

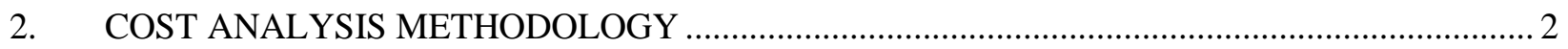

$2.1 \quad$ Work Process Step Cost ............................................................................................. 2

2.2 Technology Cost Saving Per Work Process Step............................................................... 4

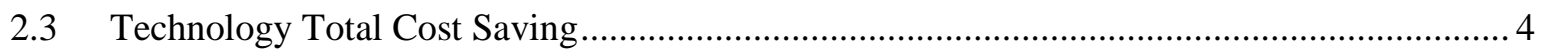

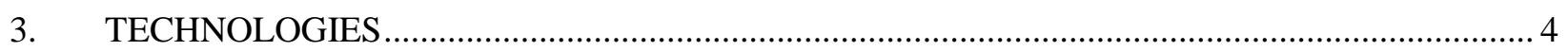

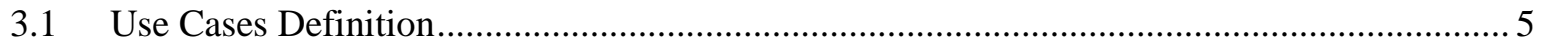

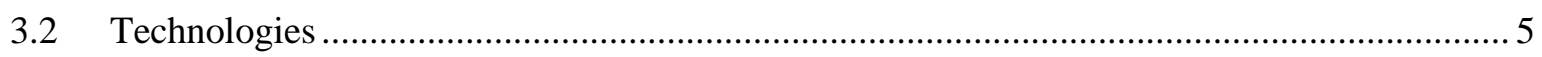

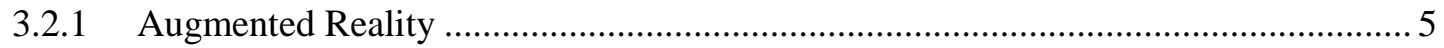

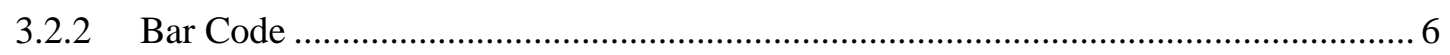

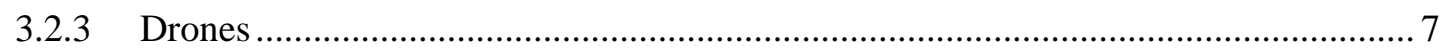

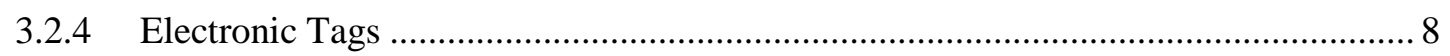

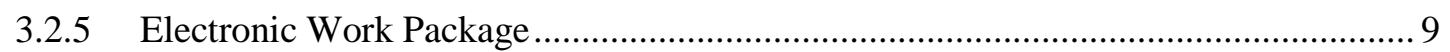

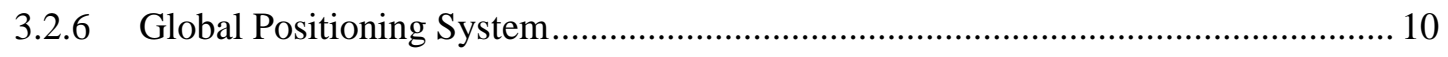

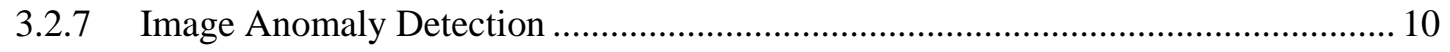

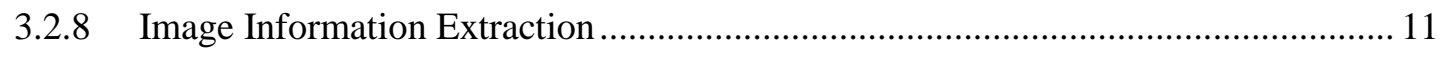

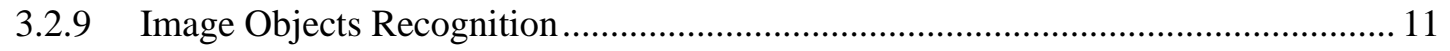

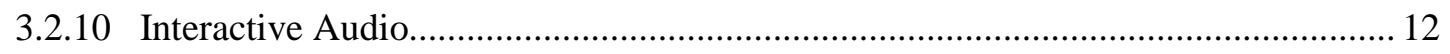

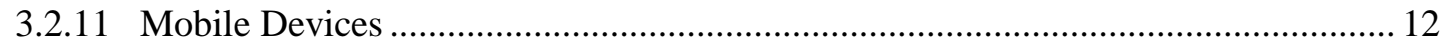

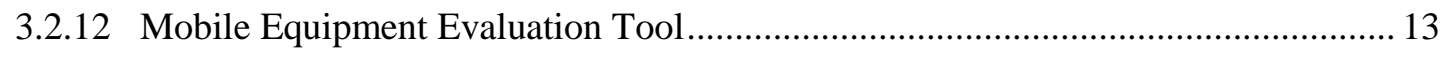

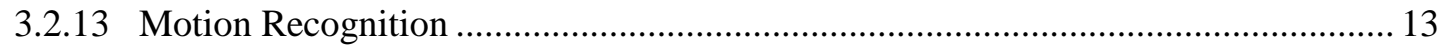

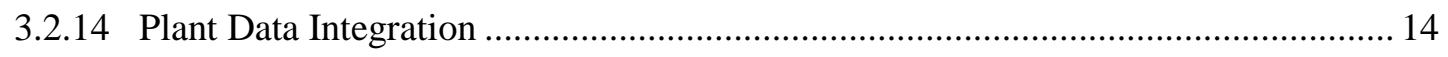

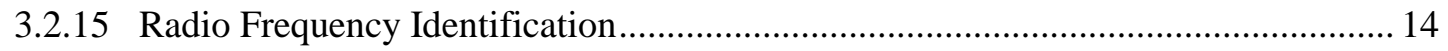

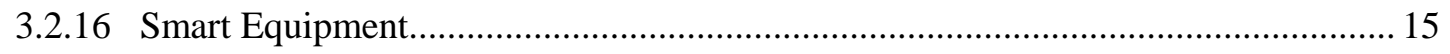

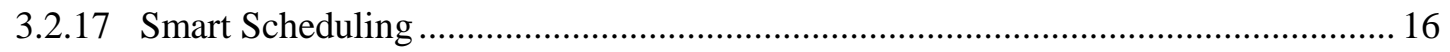

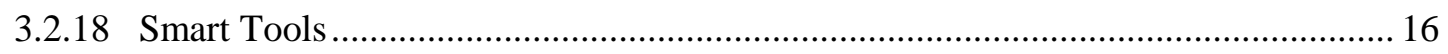

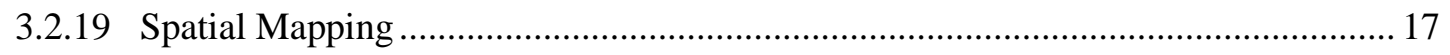

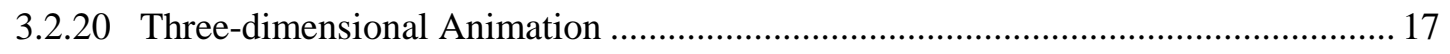

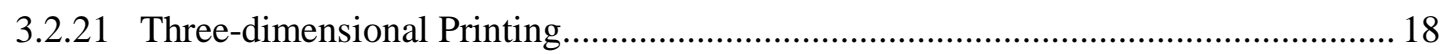

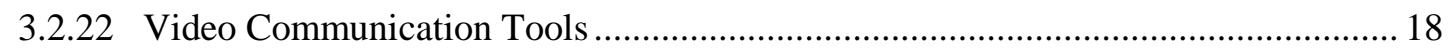

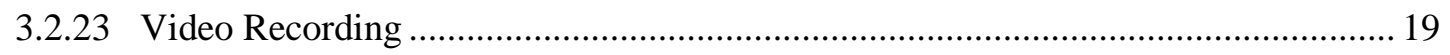

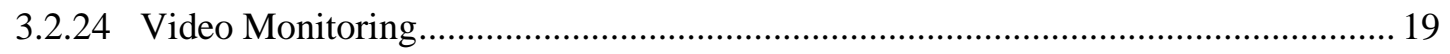

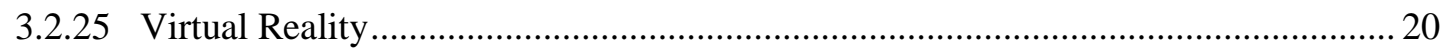

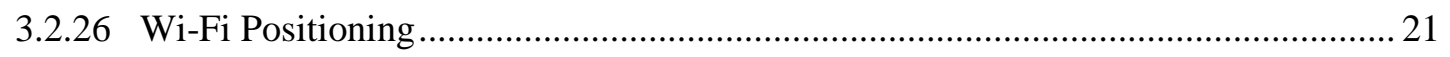

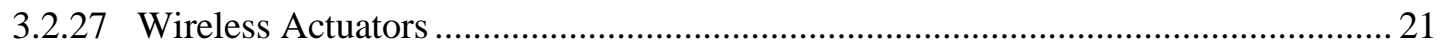




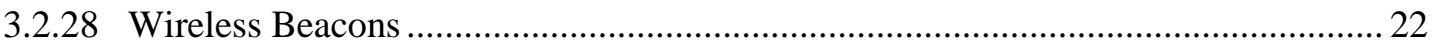

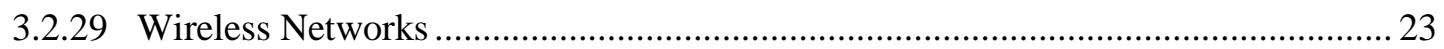

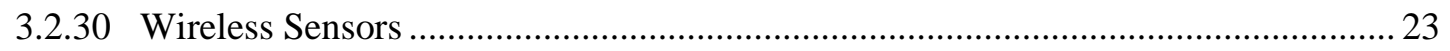

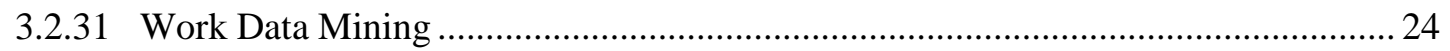

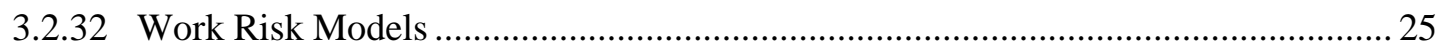

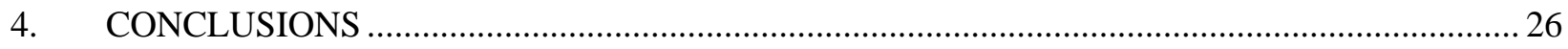

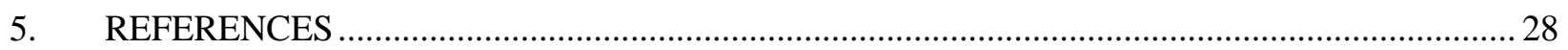

FIGURES

Figure 1. Viewing a 3-D model in the CAVE (Hugo and Slay 2016).................................................21

Figure 2. Bluetooth-enabled microcontrollers used as beacons ( $\mathrm{Al}$ Rashdan et al. 2017).......................23

\section{TABLES}

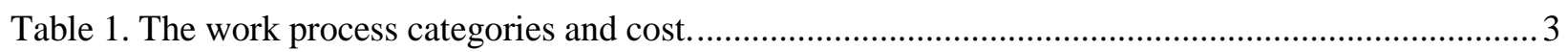

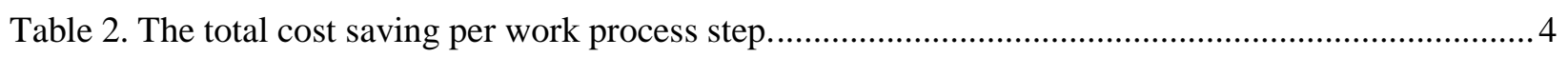

Table 3. Summary of technologies maturity and cost impact.............................................................22 


\section{ACRONYMS}

3D three dimensional

AR augmented reality

EPRI Electric Power Research Institute

eWP electronic work packages

GPS Global Positioning System

INPO Institute of Nuclear Power Operations

MT\&E materials, tools, and equipment

NEI Nuclear Energy Institute

NPP Nuclear power plant

O\&M operations and maintenance

PRA Probabilistic Risk Assessment

U.S. United States

VR virtual reality 


\section{Automation Technologies Impact on the Work Process of Nuclear Power Plants}

\section{INTRODUCTION}

Nuclear power plants (NPPs) in the United States (U.S.) are challenged with sustaining an economically profitable profile due to the plants' running cost and an increasingly competitive market landscape. Out of this cost, the operations and maintenance (O\&M) cost is around twice the cost of fuel (World Nuclear Association 2017). NPPs have a particularly high burden in managing O\&M costs. The nuclear energy industry is regulated at a significantly higher ratio than other industries; this leads to increased staffing costs. This is reflected through the work process of NPPs.

The work process is a large effort that involves many different departments across an NPP. Executing the work is extremely labor intensive. The work process in NPPs incudes a systematic set of tasks sorted by their nature, chronological order, and contributors. The majority of work in NPPs is performed using work packages, which are the primary tools of the work process, and are classically aggregations of documents needed to perform industrial maintenance. In particular, these packages include everything from forms and procedures to engineering drawings. Over decades, the work process in NPPs grew exponentially, to the extent that it became one of the main financial burdens of the nuclear power industry. As a result, the industry has recently pivoted to implementing various automation technologies, with an initial focus on electronic work packages (eWPs).

eWPs automated multiple parts of the work process (EPRI 2015). As the industry rolled-out eWPs (Shaw 2015), it realized that the majority of work processes remained and could not be automated. Guidelines were created to assist the industry in identifying strategic optimizations that affect O\&M costs (International Atomic Energy Agency 2006, EPRI 2007), or to support optimization of specific parts of the work process. These improvements in practices are inevitable; however, utilities need to be proactive to avoid a burden of growing market pressures. This research effort focused on assessing work-process components that are particularly burdensome for NPPs. Maintenance processes vary dramatically across organizations, utilities, and job scope. These variations allow for flexibility for NPPs to generate solutions that fit their needs and unique circumstances.

Automation technologies similar to the work done by Al Rashdan et al. (2018) are the means for nuclear to become a proactive industry. The aim of this report is to identify proactive automation technologies that can make significant reduction to the NPP O\&M cost. By working with industry and through strategic discussions, commonalities among various organizations were used to determine the work-process areas in which the automation technologies would have the greatest impact at reducing costs industrywide. These technologies reduce labor activities, improve capacity and uptime, and ensure the work can be completed safely. The key scope for this report is the work process in normal operations of the plant. However, due to the similarities to outage work processes, the technologies identified can be applied to the outage work process as well, and in some cases, are more suitable for outage than normal work processes. 


\section{COST ANALYSIS METHODOLOGY}

The cost analysis of this effort was performed through three parts. The first part determines the current cost of work-process steps and is shown in Section 2.1. This is a cost estimate, based on the current practices of the industry. The second part describes the mechanism to estimate the cost saving of the technology per work step and is shown in Section 2.2. The last part, shown in Section 2.3, described how the findings from Sections 2.1 and 2.2 were combined to create the overall cost saving for the technology.

\subsection{Work Process Step Cost}

To analyze the work-process cost, it was necessary to classify the work-process steps by phase or nature. The phase approach was followed by Institute of Nuclear Power Operations (INPO 2010). INPO classifies work process steps into screening, scoping, planning, scheduling and coordination, execution, and post-workweek analysis. This approach was followed in the Nuclear Energy Institute (NEI) Efficiency Bulletin (Nuclear Energy Institute 2017). NEI classifies work process steps into screening or routine/recurring maintenance, scoping and schedule, execution, and post-workweek analysis. The approach taken in this work was to classify work process steps by the nature of the task along four categories: analytical, coordination, physical, and administrative (Table 1). This classification of workprocess steps enabled easier allocation of cost to work-process step. The definition of each category is:

- Administrative. Administrative tasks are defined as requiring little to no cognitive operations to execute successfully and, therefore, present a direct path for automation technologies to enhance organization's cost breakdowns by removing tasks that do not require significant levels of human intelligence to perform. Examples of these tasks are work-package archiving, review of checklists for safety, or assignment of resources that do not require balancing priorities.

- Coordination. Coordination tasks are characterized by coordinating individuals within or across organizational boundaries to complete work tasks. In a work-package process, common coordination tasks could be planners interacting with system engineers or supervisors in the assembly of the work package or handoffs between different staff in the performance of work. This category was shown to be a large source of cost due to competing prioritization or challenging organizational communication.

- Physical. Physical tasks are characterized by the performance of physical tasks such as procedure execution, tag outs hanging, or equipment collection. Automation technologies can assist a human to perform these tasks in some cases or fully replace the human element in others.

- Analytical. Analytical tasks are those tasks that require human intelligence and problem-solving skills to execute successfully. Scoping and review or allocation of varying levels of skilled-labor resources are examples.

These categories were used to generate the results in Table 1 . Table 1 also includes the estimated cost of the steps of the work process. This estimate was found to vary from one plant to another depending on the level of process efficiency implemented at the plant. As a result, the cost presented in Table 1 is an estimate that was used for the remainder of this effort and does not constitute an industry standard. 
Table 1. The work process categories and cost.

\begin{tabular}{|c|c|c|}
\hline Work Process Step & Category of action & Cost Per Work Package \\
\hline \multicolumn{3}{|c|}{ Condition Reported } \\
\hline Review and scope & Analytical and coordination & Medium \\
\hline \multicolumn{3}{|c|}{ Work Requested } \\
\hline $\begin{array}{l}\text { Planner preparation \& } \\
\text { information collection }\end{array}$ & Analytical and coordination & High \\
\hline Initial walk down & Analytical and Physical & Medium \\
\hline Parts Identification & Analytical & Low \\
\hline Clearance order creation & Analytical & Medium \\
\hline Work Package Assembly & Administrative & Medium \\
\hline Documents verification & Administrative & Low \\
\hline \multicolumn{3}{|c|}{ Work Package Ready } \\
\hline Procurement & Administrative & Medium \\
\hline Scheduling & Analytical and coordination & High \\
\hline Crafts assignment & Analytical and coordination & Low \\
\hline Walk down & Analytical and Physical & Medium \\
\hline Supervisor pre-work review & Analytical & Medium \\
\hline \multicolumn{3}{|c|}{ Pre-work Completed } \\
\hline $\begin{array}{l}\text { Clearance order approval, } \\
\text { briefing, and preparation }\end{array}$ & $\begin{array}{l}\text { Analytical, coordination, and } \\
\text { physical }\end{array}$ & High \\
\hline Pre-job brief & Coordination & Medium \\
\hline MT\&E collection & Physical & Low \\
\hline Procedure execution & Physical and Coordination & High \\
\hline \multicolumn{3}{|c|}{ Work Completed } \\
\hline Clearance order release & $\begin{array}{l}\text { Analytical, coordination, and } \\
\text { physical }\end{array}$ & Medium \\
\hline Post maintenance testing & Physical & Medium \\
\hline MT\&E return \& cleanup & Physical & Low \\
\hline Supervisor Post-Work Review & Analytical & Low \\
\hline Final quality control review & Administrative & Low \\
\hline Work package archival & Administrative & Medium \\
\hline \multicolumn{3}{|c|}{ Work Package Closed } \\
\hline
\end{tabular}




\subsection{Technology Cost Saving Per Work Process Step}

The method used to determine the cost saving per work-process step utilizes a dual-perspective approach to cost understanding and analysis. The approach uses information obtained from literature, interviews, products, and media to identify a technology's cost impact on each step of the work package and the ratio of applicable work packages for that technology. The cost per work package is described using the execution of the work-package procedure as the reference metric. For example, a cost saving of $50 \%$ implies a technology reduces the work-package execution cost by $50 \%$ of the cost of executing the procedure. For the purpose of the report, the total cost was found using the categories defined in Table 2.

Table 2. The total cost saving per work process step.

\begin{tabular}{|c|c|c|}
\hline $\begin{array}{c}\text { Cost Saving } \\
\text { per Work Package }\end{array}$ & $\begin{array}{c}\text { Ratio of Applicable } \\
\text { Work Package }\end{array}$ & $\begin{array}{c}\text { Total } \\
\text { Cost Saving }\end{array}$ \\
\hline High & High & High \\
\hline Medium & High & High \\
\hline Low & High & Medium \\
\hline High & Medium & High \\
\hline Medium & Medium & Medium \\
\hline Low & Medium & Medium \\
\hline High & Low & Low \\
\hline Medium & Low & Low \\
\hline Low & Low & Low \\
\hline $\begin{array}{l}\text { Cost saving per work package: } \quad \text { Low }<20 \% \text { Medium 20-70\% High }>70 \% \text { of procedure execution cost } \\
\text { Ratio of applicable work package: Low }<20 \% \text { Medium 20-70\% High }>70 \% \text { of total number of work packages }\end{array}$ \\
\hline \multicolumn{2}{|}{}
\end{tabular}

\subsection{Technology Total Cost Saving}

This step combined the results from Section 2.1 and 2.2. The process used to estimate the total cost saving per technology is to:

1. Determine the impacted steps of the work process (in the first column of Table 1) by the technology deployment.

2. Determine, using the third column of Table 1, the cost of the impacted work-process steps. This represents the maximum cost saving that can be achieved if that step was eliminated.

3. Determine the total cost saving of the technology per work step using the method of Section 2.2 and Table 2.

4. Use Step 2 to scale down the results from Step 3. For example, if the cost saving from Step 3 was determined to be high, but was found to apply to work process steps that have low cost (in Table 1), the total cost saving would be low, because that is the maximum cost saving that can be achieved.

5. Average the cost saving of all work steps from Step 4 to create a holistic cost saving conclusion of implementing the technology.

\section{TECHNOLOGIES}

In order to simplify the technology applications, a set of use cases is used throughout the report. The following sections describe the identified technologies, the use cases impacted by the technology, the total cost saving from applying the technology using the analysis described in the previous section, and the maturity of the technology. 


\subsection{Use Cases Definition}

The definition for use cases groups the technology implementation into a handful of categories defined as follow:

- Location identification: Technologies that enable or enhance the organization's ability to locate resources in the plant in an automated form.

- Object identification: Technologies that automate human comprehension tasks to classify particular pieces of materials, tools, and equipment (MT\&E) or identify people and plant spatial locations.

- Improved decision making: Technologies that can assist in performing an improved decision by providing more information to key parts of the work process.

- Actions and events identification: Technologies that identify dynamic state changes, deviant humanor equipment-behavior patterns, or out-of-norm behavior that would trigger an event.

- Localizing activities: Technologies that allow for remote interactions, supervision, approval, training, or other informational exchange.

- Autonomous physical activities: Technologies that replace human physical actions. This includes remotely controlled actions and fully autonomous actions that requires no human interaction.

- Performance enhancement: Technologies to enhance the quality, speed, and safety of an activity they are applied to. These technologies include human-factors technologies to reduce the human error.

The application of the use cases are not exclusive. Due to the advanced nature of the technologies discussed, many implementations are flexible enough to affect different areas of the work process. Because of this, there may be some overlap in the particular technology's use case descriptions as their practical applications and impact on the work process are described.

\subsection{Technologies}

This section describes the automation technologies that have been considered as part of this work. The topics that are addressed for each technology are the technology description, the technology's current applications, technology's maturity level, the potential uses for the technology in the nuclear industry, and the technology's cost impact on the work process.

\subsubsection{Augmented Reality}

Augmented reality (AR) can provide specialized feedback and information access to the operators as an overlay to the operator's environment. AR information is typically displayed in some form of headset or glasses, such as Microsoft's HoloLens, Google Glass, and Magic Leap systems (Levy, 2017), to overlay the actual physical environment, but also can be displayed as an overlay of a live or recorded video stream. This information from the AR system can help to guide actions or provide more information, e.g., overlaying engineering drawings over the equipment for easy reference. AR is often applied to interact with digital objects or representations as though they were physically present in the environment. This is done if AR is coupled with location and object-identification technologies (described later). For example, as a person or video stream turns, AR would capture the objects in the newly seen environment.

While AR is mature, its deployment tools are in development. AR can be applied to a video stream on tablet or phone. If AR glasses are used, the AR interactions must be coupled with an audio interactive tool, (described later) to enable touch-free interactions. The glasses need to be regularly charged and coupled to a processing unit. AR is mostly used for entertainment applications, but is finding its way as a human assistance tool in various industries. In the nuclear industry, AR can be used for: 
- Improved decision making. AR allows users to access an overlay of information on the physical world that they perceive. For example, AR allows maintenance staff to access plant process data during walk downs, dynamically identify hazard areas, add object and equipment information to walk downs.

- Localizing activities. AR brings information that is not available to the user. It performs this by adding informational overlays, such as drawings, diagrams, or interactive information sources, that can be visually perceived by staff and enable more insight into the work task that would otherwise require collecting the information or traveling to another location of the facility to gain the same information. These tasks are especially applicable during walk downs, preparation, and procedure execution.

- Performance enhancement. AR has the potential to enhance the performance of work process tasks. Access to the informational overlays and interactive options, including procedures within an AR, can improve speed of execution, reduces human errors, map hazards and barriers, and add visual alarms , particularly in walk downs, preparations, collecting MT\&E, safety review, and procedure execution. As a rule, proper implementation of an AR system into these tasks will increase the accuracy, thoroughness, and success of these tasks.

Maturity Level: Demonstration/deployment of the core AR capabilities and development of tools to improve the human interaction and automate the creation of AR overlays.

Cost Saving: Medium.

\subsubsection{Bar Code}

Bar codes are optical identification patterns that are scanned using visual or laser scanners. A phone or tablet camera or a dedicated scanner can be used to scan a bar code. Bar codes are currently widely used in retail and logistics applications. In the nuclear industry, bar codes can be used for:

- Location identification. Bar codes applied to plant equipment and locations can assist in location identification during walk down, operations preparation, procedure execution, and other parts of the work process. Bar codes can be used to perform check-ins at locations or equipment to ensure the correct location is being assigned to a work package or found during a walk down.

- Object identification. Object identification is the primary area where bar codes can be of use. Most items have bar codes in warehousing to assist in verification tasks. Similarly, within a plant, bar codes on MT\&E can be scanned into an electronic process tracker or an eWP to perform verification of correct MT\&E use. Bar codes can also be applied on documentation, drawings, or tag outs.

- Performance enhancement. The verification use of bar codes can reduce error and enhance the performance of tasks in the work process, such as walk downs, identifying parts, collecting MT\&E, procedure execution, and more. Requiring the scan of bar codes for all locations, equipment, and parts can reduce rework situations due to misidentification and enhance performance and efficient work due to the correct objects being used at every stage. Verification at a critical step reduces the possibility of an unsafe condition occurring during execution and can assist quality-control actions by providing identifiers for all work components within the work package.

Maturity Level: Demonstration/deployment

Cost Saving: Low 


\subsubsection{Drones}

Drones are remotely controlled or autonomous vehicles that range in size from a few inches to a few feet and can operate hundreds of feet from their control unit or operate out of range based on a preconfigured program. The drone term is mostly associated with vehicles that can fly hundreds of feet above ground. However, driving or walking land robots and floating or diving water vehicles are also included in the definition of drones in this report. As a technology, drones have matured to the level that they are finding multiple applications in industry, especially in visual inspections for entertainment, security, and maintenance purposes. Drones are often used as autonomous machines with programmable instructions to perform physical actions that may be unsafe or inefficient for humans to perform. For example, drones can be used to inspect windmill blades and therefore eliminate the need for a technician to climb the windmill and visually inspect them. Advancements in drones have begun to make gains in the nuclear industry in areas of waste removal and high-hazard work in the UK and, in Japan, as part of the Sellafield and Fukushima cleanup efforts (Southwest Research Institute 2018). Using drones for performing actions like probing a certain part is a research, development, or mature topic, depending on the application. For example, some retailers are actively researching drone uses for package delivery (Kimchi et al. 2017).

Drones have limitations that need to be overcome if they are to be widely used in the nuclear industry. Dedicated flying zones need to be established for flying drones, or walking paths need to be allocated for land drones. For both cases, regulations and safety measures have to be taken to ensure the deployment would not introduce any risks to the plant. It is therefore projected that drone applications would be mostly focused on outage activities in the near future. In the context of the nuclear power industry, drones can be used for:

- Improved decision making. Because drones can enter areas that humans cannot, drones can provide information that would otherwise not be accessible to stakeholders. Drones can also perform field verifications of work completion or plant states and provide sensor data supporting those tasks for operations preparation, reviewing hazards in the plant, and quality-control duties, for example. Drones equipped with sensors, cameras, and other collection tools can be placed on a set path to gain data in areas of concern and report anomalies to a centralized data collection.

- Actions and events tracking. Drones on field duty can be constantly taking measurements and recording video or audio to compare to baseline values and alert necessary departments in the event of a deviance from normal operating states. Any anomalies found can provide valuable information for use during work process steps such as operations preparations, and walk downs.

- Localizing activities. Drone collection of video, sensor data, and other useful information can be used to enable supervisors, schedulers, and operations to remotely verify work progress and completion, and thereby perform plant activities without deploying staff to the area.

- Autonomous physical activities. Drones can be used to replace humans in physical task performance for a set of applications. Drones can be used to perform manual actions such as valves alignment. They can conduct periodic surveys such as fire loading (i.e., amount of transient combustibles). They can be outfitted with tooling, sensors, and other resilient materials in order to enter areas that are hazardous to humans to perform inspections or work. They can perform operator rounds and operator field actions, look for anomalies and events such as conducting fire watch, transport resources (such as MT\&E or documents) to and from a work site, and survey radiation field. They can complement human activities to speed up delayed tasks, compensate for lack of certain human skills, and perform actions in human-restricted areas that would otherwise be done in outage only. 
- Performance enhancement. Drones can enhance performance in nearly every work-process step. They speed up the execution of tasks, remove human error, and acts as a resource to the work process, thereby improving work-process efficiency. Drones can also replace human operators in hazardous areas. Drones can be constructed from resilient materials that can sustain high heat, dust, or radiation environments and perform data collection or simple manual tasks in such hazardous conditions.

Maturity Level: Demonstration/deployment of visual and basic tasks and development of drones to perform specific actions and take prop-based measurements

\section{Cost Saving: High}

\subsubsection{Electronic Tags}

Tag-out and tag-in processes are part of the operations clearance order for work to be safely executed. This could be as simple as installing a paper label indicating that equipment should not be operated due to certain work being performed on it or on related equipment. It could also include locks with keys that are safely kept by operations. Electronic tags are tags that perform both functions: they lock the equipment electronically and can transmit the tag status wirelessly to a plant-centralized location for tracking. Electronic tags can detect tampering and report immediately any change of status of the tag or of an equipment that is tagged out. This type of tag has been pursued for use in multiple industries (e.g., NAVSEA 2013). In the context of the nuclear power industry, electronic tags can be used for:

- Location identification. Electronic tags installed on specific equipment can assist in locating that equipment by using tag alarms, lighting methods, or other means of feedback to the O\&M staff. Electronic tags can be operated from the control room to guide staff to locations. These electronic tags can also call back to operations if an issue occurs and can communicate location data at that time.

- Object identification. Objects that have electronic tags installed can be enabled to identify themselves or respond to other stimuli to assist with identifying a particular object or piece of equipment.

- Improved decision making. Electronic tags can assist operations in making decisions by transmitting information showing the tag was tampered with or is malfunctioning. In addition, maintenance staff can benefit from additional information that an electronic tag can provide, such as ensuring tag outs and that the equipment is safe to work on.

- Actions and events identification. An electronic tag that is not operating correctly, or that has been tampered with in some way (e.g., by an individual attempting to work on incorrect equipment) can alert operations.

- Localizing activities. Fixed electronic tags on equipment eliminate the need for operations to locally check the tag out of an equipment and thereby enables the activity to be performed remotely.

- Autonomous physical activities. Typically, tags need to be hung physically by operations staff. However, electronic tags automate this process by remote actuation and enable the tagging out of equipment from the control room.

- Performance enhancement. Electronic tags can remotely and holistically provide operations with the overall status of certain equipment in the plant to reduce human errors that can result in safety concerns. They ensure the work is not performed on equipment which is not safe to work on or that can affect other plant components' safe condition.

Maturity Level: Demonstration/deployment

Cost Saving: Low 


\subsubsection{Electronic Work Package}

Electronic work packages exist across a spectrum of varying feature solutions. In a simple form, eWPs serve to replace paper work packages with an electronic version using smart or fillable pdfs. As eWPs get more advanced, they utilize extended markup language (XML) or Java-Script Object Notation (JSON) packages that handle information transfer across systems and presentation within some computerbased graphical user interfaces to fully integrate computer-based procedures (Oxstrand et al. 2015). In nearly every instance of the work process, eWPs improve the cost position of the O\&M. A list of potential functions of eWP systems and user interviews can be found in Oxstrand (2017). In the context of the nuclear power industry, eWP benefits can be found in EPRI (2015). Though the nuclear power industry has been deploying limited versions of electronic work function, this report assumes the high-end eWPs are deployed. Currently, eWPs require research and development to automate the conversion process of basic forms of eWP to an object oriented form. This enables an intelligent and adaptive forms of eWPs to be developed. eWPs can be used for:

- Object identification. eWP systems can assist in object identification particularly for documents. Documents can change frequently, and ensuring the most current revision is being performed remains particularly difficult in some organizations, especially if paper processes are still frequently used.

- Improved decision making. Coupled with data mining and data-integration technologies, eWPs provide an application interface to access and interface with data across the organization. Also, eWPs enable tracking work progress by logging the time required for step execution. This can improve scheduling, streamline operation activities, such as clearance and preparation and MT\&E collection activities.

- Localizing activities. eWPs provide access to distributed data sets and components across the work process. Rather than having physical meetings to coordinate information or approvals, disparate groups can have steps in the workflow by which they can insert or interact with information in the work package that may otherwise require physical meetings. It can also improve the supervisor and quality review efficiency by omitting irrelevant parts of the work package and enabling a digital workflow for those processes.

- Autonomous physical activities. Work-package assembly was identified as a particularly timeconsuming process in organizations that still use a paper process for any part of the work process. eWPs automate that process by allowing the use of digital templates, eliminating paper assembly and archiving, integrating recommended work-package steps automatically, and routing to the appropriate departments for approvals and information.

- Performance enhancement. Most steps in the work process are performed more efficiently using eWPs. The minimized opportunity for errors, access to plant data, optimization of procedure execution by eliminating unneeded steps and dynamically navigating through steps, and assurance of the validity of inserted information are examples of eWP benefits. By ensuring the validity of the documents, clearances, hazard review, parts, and equipment, the work is executed in a safer manner due to the minimization of the risk that improper resources can cause.

Maturity Level: Demonstration/deployment of the base form of eWP, and development of more intelligent and adaptive forms of eWPs and of methods of automated conversion.

Cost Saving: High 


\subsubsection{Global Positioning System}

The global positioning system (GPS) is a satellite triangulation technology to attain pinpoint position of equipment, devices, or people. Typical accuracy of GPS location points are within several feet. The technology is currently implemented in many types of mobile devices, in addition to dedicated GPS beacons. The use of GPS is commonly applied in navigation and location identification. GPS beacons are used to track high-value assets, especially those that are usually more expensive if they are to broadcast their location back to a central unit for tracking. If used in mobile devices, the GPS module energy consumption increases the need for device recharge. In contrast, GPS beacons require frequent battery replacement. All types of GPS-enabled devices require direct line of sight with the sky for accurate location prediction. In the context of the nuclear power industry, GPS can be used for:

- Location identification. GPS's primary addition to the work process is to assist in guiding users to specific locations. Guiding staff to the correct area of the facility for walk downs, and work execution are examples where this technology can be used. This is especially useful in less common work areas to ensure maintenance staff enter the correct area to perform their work.

- Object identification. Expensive or important mobile assets can be tagged with GPS to enable tracking equipment, components, or other items. This reduces the time needed to find the equipment, and improves its utilization in the work process.

- Improved decision making. GPS can be used by scheduling to track activities progress. This can be applied to mobile devices to track human resources and the expensive or important assets that are tagged with GPS capabilities.

- Performance enhancement. GPS impacts all activities where a physical location or object needs to be identified. It reduces human errors resulting from working on the wrong equipment or location. The GPS location can also trigger actions on mobile devices when the devices are in a certain GPS location.

Maturity Level: Demonstration/deployment

\section{Cost Saving: Low}

\subsubsection{Image Anomaly Detection}

The use of image processing for detecting anomalies can be achieved by establishing a baseline for optical, thermal, or infrared profiles of a process and capturing deviations from that baseline. The deviation from norm is a first indicator of an anomaly, but does not identify what the anomaly is. For example, if image anomaly is used for security purposes, a passing animal might indicate an anomaly, even though it is not really a security concern. This emphasizes the need for further research into methods to assist the classifications of specific events of interest. In the context of the nuclear power industry, image anomalies detection can be used for:

- Improved decision making. Image anomaly detection can be used to gain information on anomalies in plant equipment or areas. This information can assist system engineers and operations to identify potentially emergent states and can be used to predict possible maintenance needs. Review efforts are the most likely work tasks that could be automated by the implementation of image anomaly detection if the technology could identify state changes.

- Actions and events identification. Image anomaly detection is designed specifically to identify abnormal events or actions. The technology benefits most of the work process as anomaly details can inform scoping and reviews, walk downs, pre-work reviews, execution, quality control, and more. An abnormal event has the potential to impact all areas of a nuclear facility, so many areas have the potential to use the information for mitigation and planning. 
- Performance enhancement. All areas affected by image anomaly detection technologies were identified as having performance gains because the technology reduces human errors by detecting anomalies, improves diagnosis decision making, and derives real time alerts of changes in plant states, which has a positive impact on the safety of working in the plant.

Maturity Level: Demonstration/deployment using image change detection and development of applications specific anomaly detection.

Cost Saving: Low

\subsubsection{Image Information Extraction}

Image information-extraction technologies are able to extract information from pictures. An example of this type of technology is feature-extraction algorithms used in self-driving vehicles. For the power industry, examples could be gauge reading algorithms that extract readings from a picture and log it to automate human data-acquisition processes. The change-detection algorithm (Radke 2005) can also be used to acquire normal data once baselines are established for different states. If these techniques are to be used for the nuclear industry, application-specific algorithms must be developed. In the context of the nuclear power industry, image information extraction can be used for:

- Improved decision making. Whether continuously reading a gauge, detecting a thermal image pattern, or capturing the change of a breaker state, image information-extraction methods add information that is currently not available to the plant. It therefore impacts reviews and any task that requires taking measurements within the plant.

- Autonomous physical activities. Image information extraction can be used to automate processes where a human needs to acquire data from the field. This eliminates the need for staff to perform the physical activity of data acquisition.

- Performance enhancement. Image information extraction reduces the reliance on humans and thereby reduces human error. It automates the data-collection process and thus improves work efficiency, especially in scenarios where peer review is needed, in procedure execution and testing.

Maturity Level: Demonstration/deployment using image change detection for state tracking and research and development of methods to extract readings, features, additional states, and patterns.

\section{Cost Saving: Medium}

\subsubsection{Image Objects Recognition}

Image object-recognition technologies can identify objects and components in the plant. An object might be MT\&E, a part, equipment, or a location environment in the plant. This technology can look for certain shapes in a picture to correlate it to an object database or use machine learning to train an algorithm to recognize certain objects when they are seen. This technology is used by search engines to search pictures. For optimal performance, the technology needs to be customized for the specific objects of interest, which are, in this case, the common objects in NPPs. The larger the trained set, the more computational power is needed, and the slower the recognition decision making. In the context of the nuclear power industry, image object recognition can be used for:

- Location identification. Image object-recognition extraction can identify locations based on the features of the background environment. This application can improve the ability to perform walk downs, execute procedures, and perform operations tasks at the right location.

- Object identification. Image object-recognition can be used to identify parts, components, documents, or equipment in plants. By taking a picture of an object and extracting key data from the image, specific identification can be made of parts and equipment. This can be used in walk downs, MT\&E tasks, execution, and any other task that require physical identification of an object. 
- Performance enhancement. Image objects-recognition data can enhance performance of work process tasks by verifying correct equipment, parts, and MT\&E, ensuring the correct parts were ordered and delivered, and verifying correct use of tools and equipment. This reduces human-error potential and improves the safety of the plant. Object recognition can also improve the safety of the work process by identifying safety hazards as soon as they occur and reporting them.

Maturity Level: Demonstration/deployment of common objects databases and development of object-specific recognition for the nuclear industry.

\section{Cost Saving: Low}

\subsubsection{Interactive Audio}

Interactive audio can refer to audio narration and commands which can be useful in training or directing workers in particular tasks; however, it also includes more in-depth and advanced audio-system implementations that include natural language processing, answer databases, and speech recognition to truly interact with workers. Interactive audio systems may exist in some commercial-off-the-shelf solutions, but many organizations require a fully customized implementation that may require some research or development, depending on the sophistication requested by the organization. Commonplace examples of fully interactive audio systems are Apple's Siri and Amazon's Alexa. These systems operate using learning algorithms and natural language processing to understand words in sentences and ascertain what humans are asking in order to serve the proper answer (Johnson 2013). In the context of the nuclear power industry, interactive audio can be used for:

- Performance enhancement. In activities such as walk downs, operations preparations, and procedure executions, interactive audio can increase the performance of the staff by being able to give directions, commands, or information about the specific maintenance process or to respond to questions regarding area states and hazards. This communication tool enhances crafts' abilities by allowing additional flexibility and accessibility to directions or other basic information. Narration of work steps from a tablet or other mobile device in a hands-free fashion and the ability to answer basic questions can be effective at creating efficient and safe conditions for executing the work.

Maturity Level: Demonstration/deployment of common functions, and development of nuclear-industryspecific audio interactive assistant.

\section{Cost Saving: Low}

\subsubsection{Mobile Devices}

Mobile devices such as tablets and smartphones provide mobile access to information and communication tools. Smart watches are another form of mobile devices that has gained popularity recently as they provide a less intrusive form of mobile devices. Mobile devices have been deployed within the nuclear industry and are driven mostly by eWP implementation. Special tablets have been designed to comply with industrial rigidity standards (e.g., Xplore 2017). Mobile devices facilitate multiple other technologies and, therefore, have significantly more impact than their main purpose. However, the nuclear industry has various levels of reticence in regards to tablets, especially due to cybersecurity and electromagnetic interference concerns. In the context of the nuclear power industry, mobile devices can be used for:

- Location identification. By enabling GPS or Wi-Fi positioning services, the location of many mobile devices can be tracked. Coordinates within the facility can be used for tagging areas within the plant that would then upload to a mapping system for location identification.

- Object identification. Mobile devices that are equipped with cameras can perform object identification using bar codes or any of the other image-processing technologies identified in this report. 
- Improved decision making. Mobile devices allow for access to greater information and decisionmaking capabilities across the work process.

- Actions and events identification. Mobile devices can be outfitted with sensors, alarms, or connected with event databases and systems to enable input of conditions and alerts for events that are emergent. Push notifications and emergency-notification systems could be useful in maintaining awareness of plant conditions.

- Localizing activities. By their connectivity with other systems in the work process, mobile devices serve to localize information directly to the user. This ability affects many different aspects of the work process because it can connect to and facilitate transfer from most systems directly to the user.

- Performance enhancement. Similarly, the connectivity of mobile devices and the increased functionality that can be equipped to the devices increase the speed of every step of the work process. It also improves the safety of the work process by hazard mapping and the ability to alert the plant staff to unsafe issues.

Maturity Level: Demonstration/deployment

\section{Cost Saving: High}

\subsubsection{Mobile Equipment Evaluation Tool}

Mobile equipment-evaluation tools are custom tools that can encompasses multiple sensors commonly used in the nuclear power industry. These tools provides low-quality measurements and are therefore screening tools only to compliment human senses in low-risk tasks. For the nuclear power industry, an ideal tool could combine vibration, acoustic, radio, temperature, and radiation sensors. In the context of the nuclear power industry, a portable screening tool could be used for:

- Improved decision making. The ability to take direct measurements, in case of an anomaly observation, provides the plant staff with rapid additional insight into the equipment condition before creating a condition report for higher-fidelity measurements. This improves the decision-making process of the staff.

- Localizing activities. Instead of having to call in system engineers to verify an anomaly, plant staff with a tool could perform on-the-fly inspections. The portable screening tool increases reliance on secondary tools for screening low-risk tasks and thereby reduces the need to collect and return MT\&E.

- Performance enhancement. The main realized performance improvement with using a portable screening tool is due to accelerating unplanned on-the-fly inspections and detection of anomalies.

Maturity Level: Development of a mobile tool to perform multiple inspection functions that are commonly used in NPPs.

\section{Cost Saving: Medium}

\subsubsection{Motion Recognition}

Motion recognition can be used to track and comprehend human actions. Motion-processing methods coupled with machine learning could be used to enable categorization and identification of dynamic actions, such as movement and gesture. This type of intelligence has been gradually developing in other industries, especially for security applications. However, it is also finding its way into the plant work process. For example, Tang (2016) developed an algorithm on tracking craft wait time at certain tasks by tracking the craft motion using image-processing methods. In the context of the nuclear power industry, dynamic image recognition can be used for: 
- Improved decision making. Motion recognition captures data on movements, task timing, and actions. With this information, O\&M staff can gain understanding on task-performance trends and data in order to make better decisions regarding that work in the future. Tasks such as scheduling, quality control, and post-work review can all benefit from insight into the task performance and completion data.

- Actions and events identification. Motion recognition captures actions and events throughout the work process. Identifying and understanding anomalies or deviances from the norm within a nuclear facility is a critical component of the work process due to the nature of the hazards. Identifying and collecting data on these state changes can be extremely beneficial across the work process. Also, motion recognition can detect access to restricted safety or security areas to alert the craft and responsible entities.

- Performance enhancement. The insight that can be gained by capturing performance of manual physical actions by movement and gesture recognition enhances the performance of work process tasks across the life cycle of the work process.

Maturity Level: Development of tools to recognize all forms of action motions used in the nuclear power industry.

\section{Cost Saving: Low}

\subsubsection{Plant Data Integration}

Plant data integration is data consolidation to ensure data access and transfer across systems. In a typical work process of an NPP, multiple systems are used for the multiple tasks of the work process. Scheduling tools, eWPs, enterprise asset-management systems, training and human resources, and multiple other data sources in an NPP can be connected in order to provide all plant systems access to all necessary data for O\&M tasks. An example use case is an eWP using access to the training database to provide a list of qualified individuals for the work tasks to supervisors, thus preventing untrained or unqualified individuals being placed on a job. The insight provided by having the whole picture can be used for:

- Improved decision making. Plant data integration enables O\&M organizations to be able to understand the entire plant picture related to their needs. Having disparate data sources in one system enables greater insight and information of plant states for work-process steps.

- Localizing activities. Plant data integration can minimize any need for physical-information collection from plant equipment or other systems. This impacts the majority of work process steps.

- Performance enhancement. Greater access to information enables efficient decision making, reduced human errors, and faster task completion. Also, it can enhance the entire system view of plant hazards and plant status.

Maturity Level: Research and development of data-source-specific integration methods.

Cost Saving: High.

\subsubsection{Radio Frequency Identification}

Radio-frequency identification (RFID) is a technology that enables extremely small microcontrollers with antennae to harvest power from radio signal from an RFID reader and respond back to the reader with a unique identification. Due to their extremely small size and their ability to function without batteries, RFID tags provide utilities with cost-effective solutions to tracking objects, MT\&E, parts, documents, and other components of the work package process (Al Rashdan et al. 2017). The tolerance of RFID tags to extreme environments remains unclear and their ability to survive harsh working conditions is still to be developed. In the context of the nuclear power industry, RFID tags can be used for: 
- Location identification. Attaching RFID tags in specific areas is a simple way to enable the identification of locations by identifying the signals that are emitted from the RFID tag.

- Object identification. Using RFID tags for object identification can assist in ensuring the correct components are used through a process similar to checking out an item. As the RFID-tagged MT\&E pass by specific receivers, the objects will be identified, counted, and can then be filed into an eWP system for future verification. Specifically, for MT\&E collection, procedure execution, and archiving of physical documents, RFID tags can simplify those processes by automatically handling the transactions with inventory systems and foreign material exclusion zones.

- Improved decision making. RFID tagged items can be tracked throughout the plant facilities and usage data can be collected and analyzed to enhance understanding of the rate and application of usage with specific parts and components. Identification of inventory levels for procurement purposes, MT\&E collection and return, or quality-control purposes are all work-process steps that can be affected by the use of RFID tags in this way.

- Performance enhancement. RFID tags can increase work efficiency and performance by reducing the work-package loss during assembly, streamlining procurement by verifying inventory levels and transaction data, accelerating MT\&E collection and return by tracking MT\&E as they leave and are returned, and improving quality control by verification of MT\&E uses.

Maturity Level: Demonstration/deployment of commercially available RFID solutions and development of radiation and harsh environment resilient RFID tags.

Cost Saving: Medium

\subsubsection{Smart Equipment}

Modern equipment can be outfitted with sensors and advanced on-board diagnostics to automatically perform self-calibration and diagnostics (e.g., Bond et al. 2003). This can be applied to instruments (e.g., foundation field-bus instruments) or plant components (e.g., turbine-monitoring systems), and is very common in other industries, including fossil-fuel industries. The maturity of these equipment varies depending on the instrument or equipment. For example, while turbine monitoring has been deployed for multiple industries, auto-diagnosed valves are being developed (Agarwal et al. 2018). In the nuclear industry, smart equipment can be used for:

- Location identification. Smart equipment assists in the identification of location of equipment due to the ability of plant maintenance staff to communicate with it through mobile devices and ensure the correct location is identified.

- Object identification. Similar to location identification, smart equipment can interact with mobile devices used by staff to ensure that the correct object is identified prior to work starting.

- Improved decision making. Smart equipment greatly increases information provided to O\&M organizations across the work process, especially at the scoping and creation of the work package. Nearly every work-process step would be impacted by the implementation of smart equipment. Smart equipment is able to automate failure diagnosis and calibration activities, and reduce post work inspection.

- Localizing activities. Smart equipment assist in informing the scoping and review, post-work review, and execution tasks by allowing remote access to data that would otherwise need to be collected physically.

- Performance enhancement. Smart equipment augments the scoping and review steps of the work process. This reduce reliance on human decision making, reduces potential error, and increases overall process efficiency. 
Maturity Level: Demonstration or deployment of readily available smart equipment, and research and development of additional smart equipment.

Cost Saving: High

\subsubsection{Smart Scheduling}

Smart scheduling is a platform that optimally and automatically allocates schedules and resources. This is done by algorithmic or model-based advanced information processing that adaptively learns patterns and uses the gained knowledge in predicting future behavior. Smart scheduling is capable of automatically updating schedules, taking into account task prioritization, allocation and release of resources, updating resources commitments, notifying resources of their schedule, learning from resource performance for future predictions, providing review and feedback, and updating reporting options to adapt to schedule changes. In the context of the nuclear power industry, smart scheduling can be used for:

- Improved decision making. Smart scheduling learns the needed resources to execute the work from historical behavior. This provides an additional layer of information to assist in decision making across the work process. Once the system has a full understanding and model of historical states and has identified optimal intervals, parts, staff resources, and hazard issues, it can use this knowledge to enhance the schedule.

- Actions and events identification. The ability to couple the schedule with resource location and activities enables identifying actions that occurred or will occur and the source of events as they occur. Smart schedules can identify anomalous events from historical data and learn circumstances surrounding error states or abnormal conditions. This understanding can then serve to design predictive models and inform the schedule when specific conditions are more likely to occur and require mitigation. This applies to most areas of the work process.

- Performance enhancement. Smart scheduling improves resource utilization and adopts to schedule changes with optimal decisions that cannot easily be performed by a human scheduler. This improves overall work-process efficiency and accelerates activities. Smart schedules have the ability to observe more than humans, to collect data at all times, and eventually to assist in making recommendations and predicting unsafe conditions. For example, if a task takes more time than usual, this might indicate a human error.

Maturity Level: Research and development of methods to automate and optimize scheduling activities.

\section{Cost Saving: High}

\subsubsection{Smart Tools}

Smart tools are tools that have advanced functions beyond classical tools. Smart tools can communicate their measurements directly to mobile devices and verify the tool suitability for the task, communicate with wireless sensors and extract measurements on the spot, and check range and calibration information. These tool are still to be developed at this level of intelligence. In the context of the nuclear power industry, smart tools can be used for:

- Object Identification. Smart tools can identify themselves to the mobile device to make sure the tool is fit for the job, and to enable tracking of the tools.

- Improved Decision Making. Smart tools store information about their specifications, limits, calibration, and automatically ensures their fitness for the work being executed. The tools also ensure that all measurements are labeled with the location and time, and stored locally or on a server for future use, thus reduce the need for human typing information.

- Actions and Events Identification. Smart tools can identify the actions taken by the tool and report work events such as the inability of the tools to perform its task due to an equipment issue. 
- Performance Enhancement. Smart tools communicate their measurements directly and therefore reduce human input errors. They can detect when improperly used due to their ability to self-diagnose issues with their use.

Maturity Level: Development of new type of smart tools.

Cost Saving: Low

\subsubsection{Spatial Mapping}

Spatial mapping generates both a static image as well as a "step-through" series of panoramic images within the plant facilities. The mapping can be overlaid by alerts, hazard flags, or equipment to help identify areas of the plant. Spatial mapping, when fully implemented, would create a two-dimensional (2D) map as well as build a composite rendering of the visual angles of the plant. The 2D representation can be coupled with hazard logs and other informational sources so as to map areas of note in an interactive document that enables real-time examination of current equipment states and safety concerns. The mapping is usually done with $360^{\circ}$ or laser scanners.

This technology is mature and is used in other industries. A common example of this technology is Google Maps, with dual map and street-view representations to give users a simple, low-fidelity wireframe of the area and street-view images that users can click into and navigate within. An example prototype mapping of an NPP control room can be found in Hugo and Slay (2016). In the context of the nuclear power industry, spatial mapping can be used for:

- Location identification. Spatial and hazard mapping are mainly intended to give data on the location of various equipment in the facility.

- Object identification. Plant equipment, parts, and components can be mapped within the spatial map in order to allow for identification of the particular equipment needed and interaction with equipment.

- Improved decision making. Spatial and hazard mapping allows access to an interactive performance data for items. For example, within the map, a craft may need to access information about a particular generator. The craft clicks the generator and receives basic data points about the generator, its location, any recent events, or any other relevant information. This information is useful for scoping, planning, walk downs, and execution. Most areas of the work process can gain better decision-making ability and information from spatial and hazard mapping.

- Localizing activities. Spatial and hazard information can be used to localize specific areas for scoping, walk downs, and preparations. The mapping technology enables the staff to assess the equipment that would normally need to be physically assessed by sending an individual into the field.

- Performance enhancement. Spatial information can streamline walk downs and execution tasks by speeding up the process of finding the workspace and collecting information regarding plant equipment and areas. It is very useful for new staff that lack knowledge of the plant. Also, it can be used to access real-time hazard data to verify conditions within the plant.

Maturity Level: Demonstration/deployment

\section{Cost Saving: Medium}

\subsubsection{Three-dimensional Animation}

Three-dimensional (3D) animation is a computer-based 3D visualization of parts, equipment, or processes. A common engineering analogy of these animations are 3D models of components that enable exploded diagramming and allow the user to travel throughout the component (EPRI 2014). 3D animations require developing models of the component, which is time consuming if is not provided by the equipment vendor. Mapping a full system, including functioning and responsive interfaces when 
needed, can be a large undertaking. In the context of the nuclear power industry, 3D animations can be used for:

- Improved decision making. 3D animation enables the staff to virtually inspect the inside of equipment and disassemble the equipment in few clicks. This provides an additional level of information that cannot be acquired by simply looking at the equipment. 3D animation can also be used to show how MT\&E is used, demonstrate lessons learned, and simulate hazards in the workplace. 3D animation provides a familiarization and communication tool to exchange information between the work process stakeholders.

- Localizing activities. 3D animation provides an environment for work-process stakeholders to remotely exchange information that would otherwise require being present at the equipment location. It can be used to replace the real environment when work-site visits are needed in processes such as walk downs.

- Performance enhancement. 3D animation can be used for training to improve the job performance. It can visually explain the work procedure, which reduces human errors, and enable on-job training. It can also be used to demonstrate lessons learned and simulate hazards in the workplace.

Maturity Level: Demonstration/deployment

Cost Saving: Medium

\subsubsection{Three-dimensional Printing}

3D printers are multi-dimensional engraving or additive tools that can create parts made out of plastic or metal of various shapes in a matter of minutes to hours. The technology is mainly used for prototyping and temporary models use. The accuracy of 3D printing is impacted by the rate of the printing. Highaccuracy fast printers are usually large and expensive. The ability of a 3D printed part to replace an actual part is usually dependent on the materials used and the application. In the context of the nuclear power industry, 3D printing can be used for:

- Performance enhancement. 3D printing allows for the rapid creation of parts and tools for maintenance tasks. This allows for rapid iterations and improvement on plant parts. It saves procurement costs and time lost waiting for parts to be ordered. In a case in which custom MT\&E are needed for temporary but urgent solutions, $3 \mathrm{D}$ printing provides a feasible solution.

Maturity Level: Demonstration/deployment

\section{Cost Saving: Low}

\subsubsection{Video Communication Tools}

Video communication tools enable means of communication beyond standard radio or telephony systems. They can give additional depth to communications and implementation flexibility. These tools are widely used in various applications and are, therefore, ready for deployment. Overall, the cost savings from using communication tools will accrue to the additional information shared across organizations, which improves work efficiency and allows better coordination. In the nuclear industry, communication tools can be used for:

- Improved decision making. By providing more communication tools across the work process, greater information transfer between the work process stakeholders is possible. Enabling technologies like video chat can enable communication, clarification, approvals, or reporting in a visual medium that was not possible.

- Localizing activities. By using video chat, various departments can visually access the plant areas or components that would require physically traveling to that location. 
- Performance enhancement. Clarification of process steps, access to senior technicians and peers, and supervisory review and approvals are all common components of the execution of a maintenance task that can benefit from communication tools. The improved communication will lead to taskperformance improvement and better overall work efficiency.

Maturity Level: Demonstration /deployment

Cost Saving: Low

\subsubsection{Video Recording}

Video recording has been enhanced in recent years with advancements in video quality, encoding speeds, and storage options. Strategically placed units can yield benefits for recording of plant areas (Al Rashdan et al. 2017). Fixed or portable cameras, such as helmet-mounted cameras, can be used to provide a direct wide view of the work process. The technical challenge associated with using site cameras is the need to power the cameras if they are permanently mounted or to optimize their power consumption and recharge their batteries if they are to record specific tasks while powered by batteries. In the context of the nuclear power industry, video recording can be used for:

- Object identification. The video recording of parts can be used to verify the proper MT\&E was used during a review by the supervisor or quality assurance.

- Improved decision making. The benefits of video recording impact all phases of the work process. Recordings from previous jobs can be used to assist all stakeholders in exchanging information and discussing the scope. It can also become training or briefing material. Video recording can also be used as a benchmark for determining the task's required time for scheduling.

- Actions and events identification. Video recording enables comparing the plant equipment against previous running states to determine the type of actions performed during the work.

- Localizing activities. The use of video recording as a familiarization and communication tool between the work-process stakeholders eliminates the need for staff to visit the work site during steps such as walk downs.

- Performance enhancement. The video recording can be used to review and improve job quality for lessons learned. For example, it shows how MT\&E and parts perform, determines the skills required for the craft assignment, and shows the challenges associated with the execution of the task. A video recording can be used to study the risks associated with the execution of a task and to capture incidents to create lessons learned that improve the job execution in the future.

Maturity Level: Demonstration/deployment

\section{Cost Saving: Medium}

\subsubsection{Video Monitoring}

Video monitoring has many of the same characteristics and benefits of video communication tools; however, video monitoring refers to a one-way, constant stream of video. Video monitoring is extremely useful for processes seeking active insight into the performance of tasks being performed or current states of equipment or processes in the plant. Video monitoring utilizes the same cameras as video recording. However, it is more challenging to deploy due to the needed wireless infrastructure and the lower resolution of streaming, which could limit its applications. In the context of the nuclear power industry, video monitoring can be used for:

- Location identification. Video monitoring is a tool to verify the location of staff and verify the execution of work at the right location. 
- Object identification. Video monitoring is a tool to verify MT\&E being used for job execution or to verify the equipment worked on so as to ensure the work was done in the proper way and on the correct equipment.

- Improved decision making. Video monitoring allows work progress to be tracked in real time. This improves the ability to adaptively schedule the work and project progress. It also provides insight into needed resources. Video monitoring can also be used for on-the-job guidance to assist the craft in making decisions when in doubt.

- Actions and events identification. If the supervisor needs to monitor job actions to ensure they are executed correctly, video monitoring can be used to track actions executed in real time.

- Localizing activities. For tasks that require peer verification, it replaces the need for peer presence. It also enables remote and virtual participation in work-site checks such as walk downs.

- Autonomous physical activities. Video monitoring can replace supplemental work step activities that requires visual monitoring of plant processes or equipment, such as remote hazards monitoring, fire watch, and security.

- Performance enhancement. Video monitoring enables remote guidance and verification, which results in a total reduction of human error and improves safety. It helps resolve issues or obstacles rapidly and thereby improves work-process efficiency and speed. It enables an accurate projection of work progress, which enables adaptive modification to schedule and optimizes resources use.

Maturity Level: Demonstration/deployment

Cost Saving: Medium

\subsubsection{Virtual Reality}

Virtual reality (VR) is technology that enables a person to become fully immersed in a virtual representation of an environment. It is performed by wearing special headsets that shows the environment and interact with the viewer's head movement to calibrate the view of the environment. VR has been continually advancing at a rapid pace, with special momentum in the entertainment industry. Virtual reality systems can greatly enhance design and training efforts for utilities when operators or maintenance personnel can perform work tasks in a fully modeled virtual environment. Equipment can be represented in real dimensions and at scale (see Figure 1; Hugo and Slay 2016). Virtual-environment users can interact with the units or equipment in various plant areas and demonstrate or train in a no-risk environment. In the context of the nuclear power industry, VR can be used for:

- Improved decision making. VR enables the staff to virtually inspect the inside of an equipment and disassemble the equipment in a few clicks. This provides an additional level of information that cannot be acquired simply by looking at the equipment. VR can also be used to show how MT\&E is used, demonstrate lessons learned, and simulate hazards in the workplace. In terms of scheduling, VR work simulations can create a benchmark for determining a task's required time.

- Actions and events identification. VR can be used to simulate and identify craft actions in the VR environments to provide feedback prior to job execution.

- Localizing activities. VR provides an immersive environment for work-process stakeholders to remotely exchange information that would otherwise require being present at the equipment location. It can be used when work-site visits are needed in processes such as walk downs.

- Performance enhancement. VR can be used as an environment for training to improve job performance and determine skills required for a craft assignment. Human mistakes in VR have no consequences, yet provide valuable insight. VR shows lessons learned and can show MT\&E use and performance. 
Maturity Level: Demonstration/deployment, and development of interactive human-factored methods and environments for the nuclear power industry.

Cost Saving: Medium

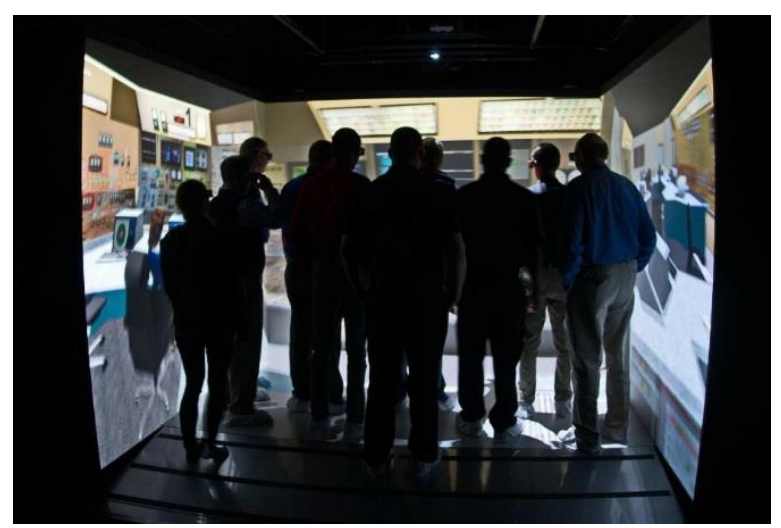

Figure 1. Viewing a 3-D model in the CAVE (Hugo and Slay 2016).

\subsubsection{Wi-Fi Positioning}

Wi-Fi positioning uses signal triangulation of Wi-Fi access points to determine locations. The technology requires a device to have a wireless transition capability and fall within at least three wireless points. It is known to have accuracy that depends on the environment of the triangulation. In the context of the nuclear power industry, Wi-Fi positioning can be used for:

- Location identification. Wi-Fi positioning enables the tracking of mobile devices in the plant to match resource location to work location for location verification, thus guiding staff to the correct area of the facility for walk downs, and work execution or collection of MT\&E.

- $\quad$ Object identification. Wi-Fi positioning, coupled with equipment-location information helps identify equipment. This is used as an additional verification step to ensure the activity or work is performed on the correct equipment.

- Improved decision making. Wi-Fi positioning can assist scheduling by enabling real-time tracking of resources locations that could feed into the schedule updates.

- Autonomous physical activities. Wi-Fi positioning can trigger actions on mobile devices when the devices are in certain locations (such as turning off wireless transmission capabilities in restricted radio areas).

- Performance enhancement. The ability to precisely define and track certain plant locations or trigger actions at these location reduces the human error associated with performing an activity at the wrong location or on the wrong equipment. Mobile devices can be set up to trigger hazard warnings when in certain triangulated locations. This enables Wi-Fi positioning to add another layer to safety administrative controls.

Maturity Level: demonstration/deployment of open space Wi-Fi positioning and development of methodology to place Wi-Fi access points for specific locations of the plant.

Cost Saving: Low

\subsubsection{Wireless Actuators}

Wireless actuators are systems that enables changing plant configuration remotely and wirelessly, thereby replacing activities that require the plant working force to manually perform plant activities. For example, manually controlled valves can be replaced or retrofitted with remote-control functions. The 
nuclear industry has a high ratio of manual actuation processes, so it is in need of this technology. However, the addition of new actuators requires ensuring compliance with regulatory requirements; this could limit the use of wireless actuators on non-safety and low-risk equipment. In the nuclear industry, wireless actuators can be used for:

- Actions and events identification. Wireless actuators enable tracking of actions performed on plant equipment.

- Localizing activities. Wireless actuators allow for the localizing of physical activities of the work process, such as operations preparation, release of the plant, and post-maintenance testing. This can be performed remotely if wireless actuators are implemented.

- Performance enhancement. Wireless actuators reduces human errors associated with field activities and significantly reduce the time needed to perform operations preparation, release of the plant, and post maintenance testing.

Maturity Level: Demonstration/deployment and of readily available wireless actuators, development of new type of actuators to control all manual activities at NPPs.

Cost Saving: Medium

\subsubsection{Wireless Beacons}

Wireless beacons, predominantly using Wi-Fi, ZigBee, or Bluetooth technologies, are batterypowered microcontrollers with unique identification that consume very low energies and are, therefore, able to run for years on a single battery (see Figure 2). These technologies have mostly been used in home automation applications, but have recently found several applications in the nuclear industry (Al Rashdan et al. 2017). The beacons' transmission strength can be configured; this enables variable range of detection and energy use. In the context of the nuclear power industry, wireless beacons can be used for:

- Location identification. Beacons can assist in guiding users to specific locations such as guiding staff to the correct area of the facility for walk downs and work execution.

- Object identification. Beacons can be coupled with equipment information to help identify equipment. This is used as an additional verification step to ensure the activity or work is performed on the correct equipment.

- Improved decision making. Beacons can assist scheduling by enabling time tracking of entry and departure from certain locations and by tracking resources used in the plant when the resources are equipped with beacons or devices to communicate with the beacons.

- Autonomous physical activities. Beacons can trigger actions on mobile devices when the devices are in range of a certain beacon (such as turning off wireless transmission capabilities in restricted radio areas).

- Performance enhancement. The ability to precisely define and track certain plant locations or trigger actions at these location reduces the human error associated with performing an activity at the wrong location or on the wrong equipment. Mobile devices can be set up to trigger hazard warnings when in proximity of certain beacons. This enables beacons to add another safety layer to administrative controls. 


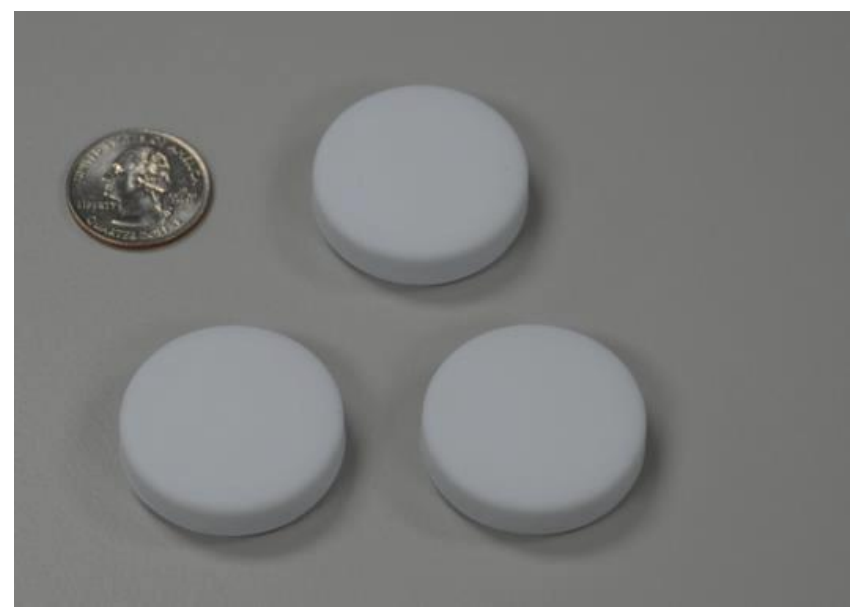

Figure 2. Bluetooth-enabled microcontrollers used as beacons (Al Rashdan et al. 2017).

Maturity Level: Demonstration/deployment

\section{Cost Saving: Low}

\subsubsection{Wireless Networks}

Wireless networks facilitate the use of many other technologies included in this report. Facility-wide Wi-Fi systems are in place at some utilities, and additional sites are increasing their wireless footprint at an increasing pace. Plants have also been exploring the use of distributed antenna systems (EPRI 2017), which are leaking cable technologies that enable targeted wireless signals in areas where Wi-Fi cannot penetrate. Because wireless networks facilitate multiple other technologies and, therefore, have much more impact than their main purpose, they are one of the highest cost-saving technologies to implement. In the context of the nuclear power industry, wireless networks can be used for:

- Location identification. Wireless networks enable the use of wireless location triangulation to track location.

- Object identification. In a similar manner to location identification, wireless networks enable the use of wireless location triangulation to identify equipment.

- Improved decision making. Wireless networks facilitate continuous and remote sensor, work process, and scheduling data access for all parts of the work process and enable the use of video communication tools and remote video applications.

- Performance enhancement. In ways similar to those described for improved decision making, wireless networks impact the performance of all parts of the work process by facilitating access to work process data in text or visual format, which increases work-process efficiency and reduces human error.

Maturity Level: Demonstration/deployment of Wi-Fi networks.

\section{Cost Saving: High}

\subsubsection{Wireless Sensors}

Wireless enabled sensors are instruments that have wireless transmission capabilities. In the context of this report, wireless sensors collect data from the various physical process parameters in a non-intrusive manner and feed that data into a central wireless processing system. They can also provide feedback on actuator actions. For example, manually controlled valves can be retrofitted with remote monitoring functions (Agarwal et al. 2018). 
Wireless sensors are useful in old plants in which cabling infrastructure does not cover all parts of the plant. The main challenge associated with wireless sensors are energy sources, which motivated research into power harvesting methods from process energy sources (Gurumurthy et al. 2013). Wireless sensors have gained increasing interest in NPPs, especially as wireless networks find their way into the nuclear industry, and advanced pattern detection methods that require additional sensors are being investigated (e.g., Fernandez 2017). In the context of the nuclear power industry, wireless sensors can be used for:

- Location identification. Wireless sensors that are capable of transmitting directly to a mobile device can track locations, especially if these devices have limited signal range, as is true of Bluetooth- or ZigBee-enabled devices.

- Object identification. Using the same concept as described for location identification, wireless sensors can be used to identify equipment.

- Improved decision making. Wireless sensors increase the data available for analysis of tasks and allows better decision making for all stakeholders, especially the systems engineer. It provides an additional layer of information that improves failure diagnosis and reduces needed inspection and calibration activities.

- Actions and events identification. The added information enabled by wireless sensors allows instantaneous detection of issues and event as they occur and enables the tracking of actions.

- Localizing activities. The ability to access sensor data remotely streamlines analysis by system engineers, preparation and information collection of planners, preparation for operations, oversight by supervisors, and execution by crafts. The data provided by wireless sensors also provide insight into work progress and thereby benefit the scheduling processes.

- Autonomous physical activities. For activities that require acquisition of readings or measurements, such as operations preparation, wireless sensors eliminate the need for an operator's presence in the field.

- Performance enhancement. Wireless sensors improve diagnosis-process efficiency and speed and reduce dependence on human decision making. They provide direct indications of plant condition and thereby enable better job preparation and inform procedure execution. They also provide direct safety benefits from safety-related sensors, such as radiation detection, and indirect benefits from detecting an improper action or system response to work.

Maturity Level: Demonstration/deployment of readily available wireless sensors and research, and development of new types of wireless sensors and power harvesting methods.

\section{Cost Saving: High}

\subsubsection{Work Data Mining}

Data mining includes computational mining and processing information from text, image files, and drawings, or other forms of data. It can apply to paper-based or electronic documentation, and can extract, through informed and data-based processes, information and patterns from documents and present the information in a more-accessible digital format to better facilitate knowledge transfer and understanding within the organization.

Data-mining technologies are rapidly advancing in multiple applications. Internet search engines are the most common data-mining application. Using data mining, it is possible to search millions of documents in fractions of a second. The extensive storage and computational power needs that are necessary to perform data mining require investment in adequate infrastructure. Mining non-textinformation, such as images or audio, requires further research and development as do mining methods that are specific to the nuclear industry ( $\mathrm{Al}$ Rashdan et al. 2016). In the nuclear industry, data mining can be used for: 
- Improved decision making. Data mining enables better decision making and information access by bringing together many forms of text- or image-based information into a readily accessible digital format. Most utilities still have paper components of processes, such as drawings, parts lists, or archived reports, that may be cumbersome to handle and find when needed. Data mining all of these information sources can allow aggregation and information input into a modern database system for easy search and use.

- Localizing activities. Using data mining, information will be localized to an enormous extent. For example, instead of having to retrieve archived work packages or engineering drawings from file cabinets or archival areas, the information would be available within the system. This would speed planning and work-package assembly processes dramatically.

- Performance enhancement. Many tasks will see performance enhancement simply by having greater access to information when needed. Scheduling and planning reviews could mine work packages or templates for changes or errors to improve their future activities. Engineering reviews would be performed faster as the engineer has historical information when needed to validate plant conditions. Pre-job briefs and walk downs would be improved by taking lessons learned from past work packages that were mined and the relevant information from which was placed in an easily accessible system. Access to previous errors or noted difficulties in past work processes would enable greater preparation and mitigation of safety concerns and greater access to information than was possible when information was stored in a purely paper form. This would allow the staff to make better decisions with regards to the work tasks and potential hazards.

Maturity Level: Research and development of methods to acquire and classify data from work process documents of the nuclear power industry.

\section{Cost Saving: High}

\subsubsection{Work Risk Models}

Probabilistic risk assessment (PRA) models are mathematical models to quantify a plant or specific process-event risk by accumulating the risk of sub-events that can lead to an event. PRA has been used extensively in the nuclear power industry, mainly for regulatory and safety-analysis purposes. Work-risk models are PRA models that quantify the risk of maintenance work by type or nature and work package, individual work steps, resource, and equipment based on historical behavior. Some form of this is applied through a human decision-making process in NPPs (Nuclear Energy Institute 2016). It is used beyond the typical regulatory compliance requirement in NPPs. In the context of the nuclear power industry, work risk models can be used for:

- Improved decision making. Work risk models can impact all steps of the work process by assessing risk states resulting from the specific work steps. They can reduce the time needed for every step of the work process by eliminating low-risk tasks or reducing the time required for executing every step of the work process to the extent reflected by the risk of error in performing that step. They can also reduce complementary activities and time spent to perform complementary low-risk activities, such as selecting a wrong tool for low-risk tasks. In contrast, they adequately increase the time needed for important or high-risk tasks.

- Actions and events identification. Work risk models serve to identify trends and make predictions on current or future states. The models can greatly inform the likelihood and risk of anomalies or deviant states in the plant. Over time and with increasing volumes of data, these models can operate in a way to predict deviant states to avoid or mitigate such events.

- Autonomous physical activity. Work risk models could eliminate physical tasks or activities, such as testing or peer review, for low-risk tasks. 
- Performance enhancement. Work risk models optimize resources use according to work-process risks and give unique information that can enable the plant to operate proactively across plant conditions and perform all tasks from a more informed position.

Maturity Level: Research and development of work risk models.

Cost Saving: High

\section{CONCLUSIONS}

This analysis of the technologies considered in this report identified multiple benefits to the work process that have direct cost-saving impact. This effort generated a list in Table 3 of technologies that fit each technology to the three cost-saving categories. The technologies that have high cost-saving impact are identified as drones, electronic work package, mobile devices, plant data integration, smart equipment, smart scheduling, wireless networks, wireless sensors, work data mining, and work risk models. The technologies that have medium cost-saving impact are identified as AR, image information extraction, mobile equipment evaluation tools, RFID, spatial mapping, 3D animation, video recording, video monitoring, VR, and wireless actuators. The technologies that have low cost-saving impact are identified as bar codes, electronic tags, image anomaly detection, image objects recognition, global positioning systems, interactive audio, motion recognition, smart tools, 3D printing, video communication tools, Wi-Fi positioning, and wireless beacons.

The effort concluded that technologies with high cost impact that requires more development and/or research into the technology as a whole or specific aspects of their application are drones, eWPs, plant data integration methods, smart equipment, smart scheduling, wireless sensors, work data mining, and work risk models. The technologies with medium cost impact that require more development and/or research into the technology as a whole or specific aspects of their application are AR, image information extraction, mobile equipment evaluation tools, radio-frequency identification, virtual reality, and wireless actuators. The technologies with low cost impact that require more development and/or research into the technology as a whole or specific aspects of their application are image anomaly detection, image objects recognition, interactive audio, motion recognition, smart tools, and Wi-Fi positing. While the focus of this study is cost saving, these technologies have benefits beyond simply cutting costs, because many will also allow for efficiency gains and improvements in safety. 
Table 3. Summary of technologies maturity and cost impact.

\begin{tabular}{|c|c|c|c|c|}
\hline \multirow[b]{2}{*}{ Technology } & \multicolumn{3}{|c|}{ Maturity } & \multirow[b]{2}{*}{$\begin{array}{c}\text { Cost } \\
\text { Impact }\end{array}$} \\
\hline & Research & Develop & $\begin{array}{c}\text { Demonstrate } \\
\text { /Deploy }\end{array}$ & \\
\hline AR & - & $X$ & $\mathrm{X}$ & Medium \\
\hline Bar code & - & - & $\mathrm{X}$ & Low \\
\hline Drones & - & $\mathrm{X}$ & $\mathrm{X}$ & High \\
\hline Electronic tags & - & - & $\mathrm{X}$ & Low \\
\hline Electronic work package & - & $X$ & $\mathrm{X}$ & High \\
\hline GPS & - & - & $\mathrm{X}$ & Low \\
\hline Image anomaly detection & - & $\mathrm{X}$ & $X$ & Low \\
\hline Image information extraction & $X$ & $\mathrm{X}$ & $X$ & Medium \\
\hline Image objects recognition & - & $\mathrm{X}$ & $X$ & Low \\
\hline Interactive audio & - & $X$ & $X$ & Low \\
\hline Mobile devices & - & - & $X$ & High \\
\hline Mobile equipment evaluation tool & - & $\mathrm{X}$ & - & Medium \\
\hline Motion recognition & - & $\mathrm{X}$ & - & Low \\
\hline Plant data integration & $\mathrm{X}$ & $\mathrm{X}$ & $\mathrm{X}$ & High \\
\hline Radio frequency Identification & - & $\mathrm{X}$ & $\mathrm{X}$ & Medium \\
\hline Smart equipment & $\mathrm{X}$ & $X$ & $\mathrm{X}$ & High \\
\hline Smart tools & - & $X$ & - & Low \\
\hline Smart scheduling & $X$ & $X$ & - & High \\
\hline Spatial mapping & - & - & $\mathrm{X}$ & Medium \\
\hline Three dimensional animation & - & - & $\mathrm{X}$ & Medium \\
\hline Three dimensional printing & - & - & $\mathrm{X}$ & Low \\
\hline Video communication tools & - & - & $\mathrm{X}$ & Low \\
\hline Video recording & - & - & $\mathrm{X}$ & Medium \\
\hline Video monitoring & - & - & $\mathrm{X}$ & Medium \\
\hline VR & - & $\mathrm{X}$ & $X$ & Medium \\
\hline Wi-Fi positing & - & $\mathrm{X}$ & $\mathrm{X}$ & Low \\
\hline Wireless actuators & - & $X$ & $X$ & Medium \\
\hline Wireless beacons & - & - & $X$ & Low \\
\hline Wireless networks & - & - & $\mathrm{X}$ & High \\
\hline Wireless sensors & $X$ & $\mathrm{X}$ & $\mathrm{X}$ & High \\
\hline Work data mining & $\mathrm{X}$ & $\mathrm{X}$ & - & High \\
\hline Work risk models & $X$ & $X$ & - & High \\
\hline
\end{tabular}




\section{REFERENCES}

Agarwal, V., Buttles, J., and Al Rashdan, A. (2018). Final CRADA Report for Enhanced and Miniaturized Wireless Valve Position Indicator Prototype, INL/EXT-18-44972, Idaho Falls: Idaho National Laboratory.

Al Rashdan, A., Oxstrand, J. and Agarwal, V., (2016). Automated Work Package: Conceptual Design and Data Architecture, INL/EXT-16-38809, Idaho Falls: Idaho National Laboratory.

Al Rashdan, A., St Germain, S., Boring, R., Ulrich, T. and Rice, B., (2017). Automated Work Packages: Radio Frequency Identification, Bluetooth Beacons, and Video Applications in the Nuclear Power Industry (INL/EXT-17-43264), Idaho Falls: Idaho National Laboratory.

Al Rashdan, A., Oxstrand, J. \& Agarwal, V. (2018) Automated work packages: Capabilities of the future. Nuclear Technology, 202:2-3, 201-209. doi: 10.1080/00295450.2017.1406774.

Bond, L. J., Jarrell, D.B., Koehler, T.M., Meador, R.J., Sisk, D.R., Hatley, D.D., Watkins, K.S. Chai, J. and Kim, Wooshik. NERI Final Project Report: On-Line Intelligent Self-Diagnostic Monitoring System for Next Generation Nuclear Power Plants. United States. doi: 10.2172/15010296.

Electric Power Research Institute (EPRI). (2007). Operations and Maintenance Development: Work Planning Assessment Guidelines for Nuclear Power Plant Personnel, EPRI-1015253. Palo Alto: EPRI.

Electric Power Research Institute (EPRI). (2014). K-Line 3-D Animated Maintenance Application Version 1.0, EPRI- 3002000163. Palo Alto: EPRI.

Electric Power Research Institute (EPRI). (2015). Improving the Execution and Productivity of Maintenance with Electronic Work Packages, EPRI-3002005363. Palo Alto: EPRI.

Electric Power Research Institute (EPRI). (2017). Use of LTE Cellular Network and Distributed Antenna Systems to Improve Connectivity and Increase Data Transfer: A Plant Monitoring Initiative, EPRI3002009128. Palo Alto: EPRI.

Fernandez, M.G., Tokuhiro, A., Welter, K., \& Wu, Q. (2017). Nuclear energy system's behavior and decision making using machine learning. Nuclear Engineering and Design, 324, 27-34.

Gurumurthy, S.R., Agarwal, V. and Sharma, A. (2013). Optimal Energy Harvesting from a High-Speed Brushless DC Generator-based Flywheel Energy Storage System. IET Electric Power Applications, 7(9), 693-700. doi: 10.1049/iet-epa.2013.0134.

Hugo, J.V. and Slay III, L., (2017). Human Factors and Modeling Methods in the Development of Control Room Modernization Concepts, INL/CON-16-40364). Idaho Falls: Idaho National Laboratory.

Institute of Nuclear Power Operations (INPO), (2010). Work Management Process Description, INPO AP-928, Rev. 5, Mat 2017. Atlanta: INPO

International Atomic Energy Agency (IAEA). (2006). Integrated Approach to Optimize Operation and Maintenance Costs for Operating Nuclear Power Plants, IAEA-TECDOC-1509. Vienna: IAEA.

Johnson, B. (2013, Feb.). How Siri Works. HowsStuffWorks. Retrieved from electronics.howstuffworks.com/gadgets/high-tech-gadgets/siri2.htm.

Kimchi, G., Buchmueller, D., Green, S.A., Beckman, B.C., Isaacs, S., Navot, A., Hensel, F., Bar-Zeev, A. and Rault, S.S.J.M., (2017). Amazon Technologies Inc. Unmanned aerial vehicle delivery system. U.S. Patent 9,573,684. Retrieved from https://patentimages.storage.googleapis.com/66/63/33/ee453e2485aa7d/US9573684.pdf 
Levy, S. (2017, Dec.). The race for AR glasses starts now. Wired, Retrieved from https://www.wired.com/story/future-of-augmented-reality-2018/.

NAVSEA, (2013), Tag-Out User's Manual, Rev. 7. S0400-AD-URM-010/TUM, NSN 0910-LP-1108193.

Nuclear Energy Institute (NEI). (2016). Efficiency Bulletin: 16-15a, Work Screening Process, EB-16-15a. Washington D.C: NEI.

Nuclear Energy Institute (NEI). (2017). Efficiency Bulletin: 17-20, Further Streamline the Work Management Process. EB-17-20, Washington D.C: NEI.

Oxstrand, J.H., Al Rashdan, A., Le Blanc, K.L., Bly, A.D. and Agarwal, V. (2015). Automated Work Packages Prototype: Initial Design, Development, and Evaluation, Light Water Reactor Sustainability Program, INL/EXT-15-35825. Idaho Falls: Idaho National Laboratory.

Oxstrand, J. (2017). Development of Utility Generic Functional Requirements for Electronic Work Packages and Computer-based Procedures, INL/CON-16-40080. Idaho Falls: Idaho National Laboratory.

Radke, R.J., Andra, S., Al-Kofahi, O. and Roysam, B. (2005). Image change detection algorithms: a systematic survey. IEEE Transactions on Image Processing, 14(3), 294-307.

Shaw, D. (2015, May-June). Electronic Work Package. Nuclear Plant Journal, 33(3). Retrieved from http://digitaleditions.nuclearplantjournal.com/MJ15/\#42.

Southwest Research Institute. (2018, March 20). SwRI-led team to develop drones for use In Fukushima Daiichi Nuclear Power Plant [Press release]. Retrieved from https://www.swri.org/press-release/swriled-team-develop-drones-use-fukushima-daiichi-nuclear-power-plant.

Tang, P., Zhang, C., Yilmaz, A., Cooke, N., Boring, R.L., Chasey, A., Vaughn, T., Jones, S., Gupta, A. and Buchanan, V. (2016). Automatic imagery data analysis for diagnosing human factors in the outage of a nuclear plant. Proceedings of the $7^{\text {th }}$ International Conference on Digital Human Modeling and Applications in Health, Safety, Ergonomics and Risk Management, Toronto, ON, CANADA, (pp. 604-615), Springer, Cham.

World Nuclear Association (2017). Nuclear Power Economics and Project Structuring: 2017 Edition (Report No. 2017/001). England and Wales: World Nuclear Association.

Xplore: PC Xplore Technologies [Xslat B10 Fully Rugged Tablet]. (2017). Retrieved from https://www.xploretech.com/downloads/Marketing_Brochures/B10/XSLATE_B10_Specsheet_ENG. pdf. 